\title{
Orthopaedic Patients Who Require Intensive Care Admission
}

\section{Surya Gandham*}

Department of Orthopaedics, Warrington Hospital, Liverpool, UK

\begin{abstract}
The purpose of this study is to evaluate the causes, numbers, demographics and outcomes of all trauma and elective patients admitted under orthopaedic care but needed intensive care input during their inpatient stay. A retrospective case note study looking at all trauma patients admitted between January 2009 and August 2010 from a University Teaching Hospital that needed ITU admission during their inpatient stay. ITU records were cross matched with an orthopaedic trauma database to create a list of patients. 43 trauma patients were admitted to ITU from orthopaedic care during this 19 month period. These patients were then split into three categories 1) Trauma patients (one fracture) 2) Poly trauma patients (<2 fractures) 3 ) Transfers (Inter-hospital transfers). Within the "trauma" patients' category, $25 \%$ of patients were admitted due to post-operative complications following hip hemi-arthroplasties, $17 \%$ due to wound debridement and soft tissue infection and $14 \%$ attributed to post-oprative complications following IMHS fixation. $30 \%$ of patients needed ITU input due to sepsis, $19 \%$ due to acute renal failure and $17 \%$ to respiratory failure. 11 patients were categorised as "poly-trauma" patients. $55 \%$ of patients sustained a RTA whilst the rest were split between "fall from height" and "crush" injuries. $34 \%$ of these patients needed ventillatory support and inotropes. The mortality rate was highest and the length of stay in ITU was longer in the "trauma" cohort, $21 \%$ and 4 days respectively.Largest proportion of ITU admissions were due to post operative complications following hip surgery especially hemi-arthroplasties. However, a surprising group that emerged was the number of patients admitted to ITU who had metal work and soft tissue infections. This has highlighted the need for pre-operative optimisation and post-operative vigilance in patients with orthopaedic infections and hip fractures.
\end{abstract}

\section{Introduction}

The critically ill surgical patient accounts for $60-70 \%$ of the workload of the general Intensive Care Units [ICUs] in the UK [1]. Current clinical opinion and available evidence suggests that the early appropriate referral of patients to ICU can significantly reduce early, and possibly late, mortality in the critically ill [2].

Trauma is classified as bodily injury severe enough to pose a threat to life or to individual parts of the body such as a limb or an organ $[3,4]$. Trauma remains a national and worldwide public health problem and is a leading cause of morbidity and mortality, especially among previously healthy, productive young people and among males in particular [3]. The majority of trauma care is carried out by orthopaedic surgeons and is a major component of their hospital practice. It is therefore important to understand the role and implementation of ICU's in practice.

As well as trauma patients, elective orthopaedic surgery represents a significant proportion of patients requiring intensive care unit admission and is also vulnerable to a wide range of complications [5]. Management of these complications requires understanding of preoperative conditions, intra-operative management and early recognition of any signs and treatment of postoperative complications [6]. These patients may be admitted to intensive care as a result of emergency surgery or elective procedures but healthcare staff must be aware of conceivable complications. Potential systemic complications include pneumonia, pulmonary embolism, sepsis or myocardial infarction whilst procedure specific or local complications such as neurovascular injuries are also possible.

Currently in the UK, there is no nationally coordinated policy for the care of the severely injured however regional trauma centres are in the process of being developed. These specific trauma centres for major injuries are designed to provide better outcomes for correctly referred patients. Overseas trauma systems, which have rationalised their service, are able to provide significantly improved healthcare and outcomes $[7,8]$. These hospitals are designated to receive patients with severe injuries and medical expertise is concentrated within $[6,7]$.
Delayed and inappropriate care pathways can lead to poor outcomes and avoidable deaths. Trauma costs the NHS on average $£ 300-400$ million per year and $£ 3$ - 4 billion are lost in societal costs [6].

The aim of this study is to evaluate the causes, numbers, demographics and outcomes of patients admitted under orthopaedic care, including trauma patients, who needed intensive care input during their inpatient stay.

\section{Method}

The Department of Trauma and Orthopaedics at Aintree University Hospital is a busy unit for both trauma and elective cases. The cohort of patients analysed for the purposes of this study were taken between January 2009 and August 2010.

A retrospective, single-centred, non-randomised observational study of all elective and trauma patients admitted to ICU during their inpatient stay was undertaken. ICU records were cross-matched with an orthopaedic database to create a list of patients. This process identified 60 patients who were admitted under orthopaedic care and subsequently developed problems which required intensive care.

31 patients were male and 29 were female with an average age of 64 . These patients were then split into four groups: [mean age/median age]

1) Elective 2) Trauma [1 fracture] 3) Poly Trauma [ $>2$ fractures] 4) Transfer [Inter hospital].

*Corresponding author: Surya Gandham, Department of Orthopaedics, Warrington Hospital, Liverpool, UK, E-mail: suryagandham@hotmail.com

Received April 10, 2013; Accepted June 26, 2013; Published June 28, 2013

Citation: Gandham S (2013) Orthopaedic Patients Who Require Intensive Care Admission. J Trauma Treat 2: 169. doi:10.4172/2167-1222.1000169

Copyright: (c) 2013 Gandham S. This is an open-access article distributed under the terms of the Creative Commons Attribution License, which permits unrestricted use, distribution, and reproduction in any medium, provided the original author and source are credited. 
Information detailing general demographics, the reason for admission and orthopaedic procedure, reason for ICU admission, ICU input, length of ICU stay and mortality were inputted into a proforma.

\section{Results}

In the Elective Surgery group, 17 patients were identified as having been transferred to ICU following their operations shown in Figure 1 , the greatest number of patients requiring ICU input were those undergoing total hip replacements $47 \%$, followed by foot and ankle procedures $24 \%$.

The main reasons for ICU admission in this cohort of patients was for close monitoring reasons $29 \%$ and cardio-vascular complications $24 \%$ (Figure 2). Once these patients were transferred to ICU they received mainly monitoring input $63 \%$ with smaller proportions receiving procedures $16 \%$ and inotropes $11 \%$ (Figure 3 ). Of the elective patients, 2 required ICU admission due to intraoperative complications whilst 15 patients suffered from postoperative complications.

The largest group of ICU admissions came from the trauma cohort of patients. This group consisted of 28 patients whom accounted for $47 \%$ of all ICU admissions. Figure 4 illustrates the orthopaedic reasons for initial admission.

Two main groups of patients can be derived from the above data: 1. Hip fracture $46 \%$ 2. Infected cases [40\%]. 13 patients undergoing hip fracture surgery were admitted to ICU with hemi-arthroplasty as the procedure with the highest risk of post operative complications requiring ICU input. 11 patients in total were admitted under

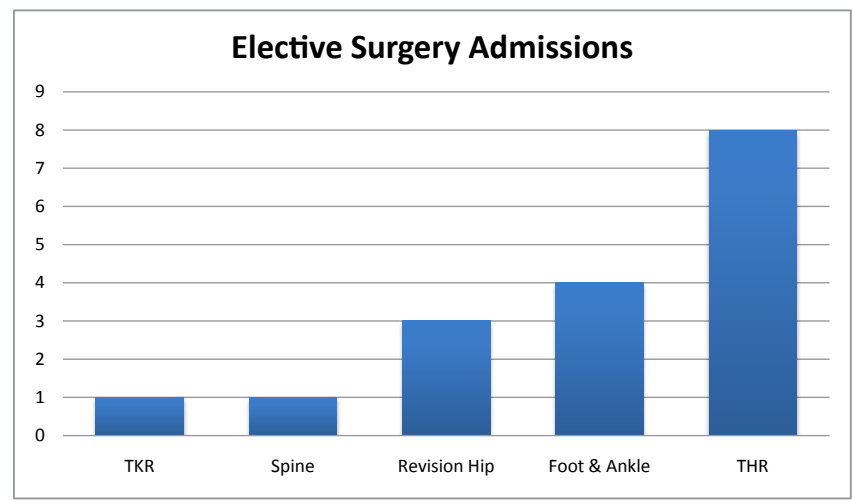

Figure 1: Graph showing the admission reasons for elective surgery

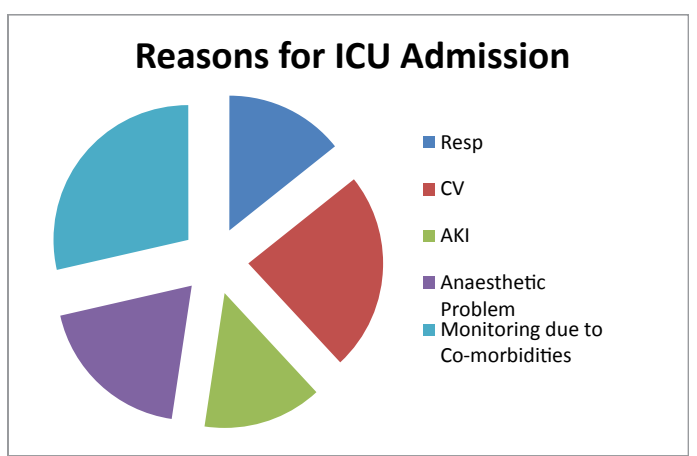

Figure 2: Chart showing the reasons for ICU Admission for patients undergoing elective surgery]

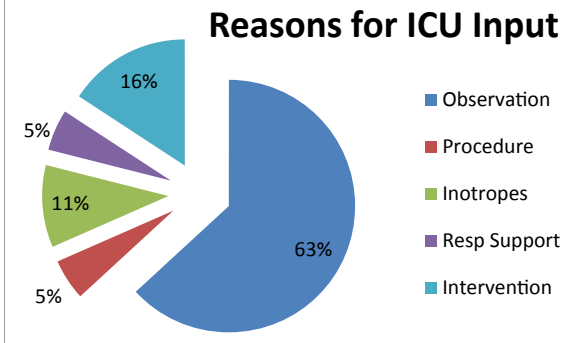

Figure 3: Chart showing the input from ICU for patients undergoing elective surgery

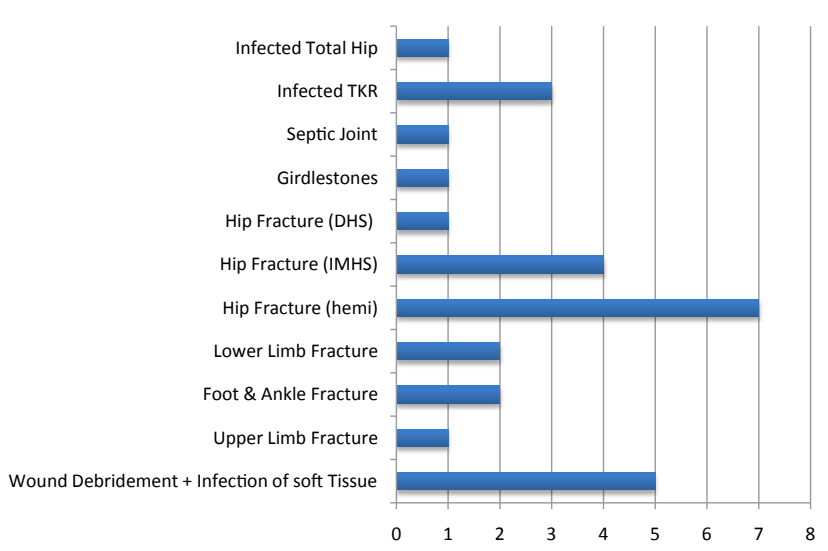

Figure 4: Chart to show orthopaedic reasons for initial admission

orthopaedics due to either infected metal work or soft tissue infections.

Infection was the primary reason [30\%] why these patients were treated on ICU (Figure 5) with antibiotics, monitoring and respiratory support as the mainstay of their treatment (Figure 6).

The poly-trauma cohort (Figure 7) contained 11 patients with 6 of those patients admitted due to road traffic accidents. They were mainly treated with interventions such as chest drains, central lines [33\%] etc and observation [25\%] (Figure 8). Of the patients that required respiratory intervention, 2 required invasive ventilation.

There were 6 patients transferred to our unit that required ICU admission. Four of which were neurosurgical emergencies and were treated firstly by the Walton Neurocentre [regional tertiary neurosurgical referral centre].

The average Length of Stay [LOS] on ICU was also calculated. Figure 9 show that the Transfers category had the longest average LOS. This result was skewed due to one neuro surgical patient staying for 102 days. Otherwise, between 2-3days was the average LOS.

Nine patients died following their admission to ICU. $67 \%$ of these patients were admitted for "Trauma" as seen in Figure 10.

\section{Discussion}

This study was undertaken to analyse the causes for ICU admission in orthopaedic patients. Work has been done focussing on general surgical patients and their need for ICU input whereas minimal literature has been published commenting on orthopaedic patients. As 


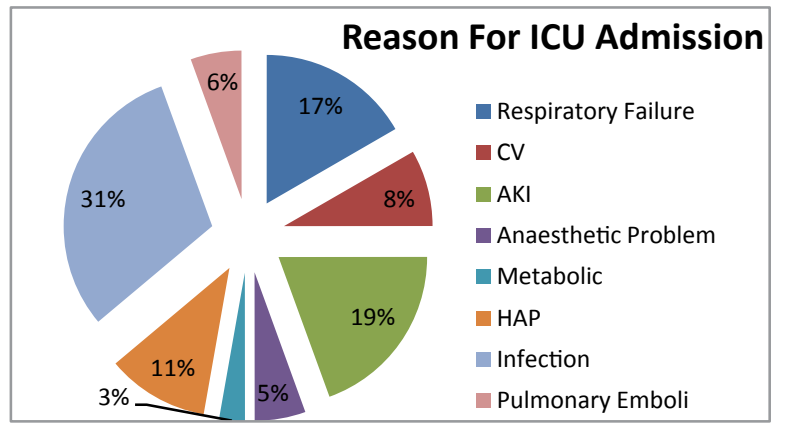

Figure 5: Chart showing the reasons why trauma patients were treated on ICU

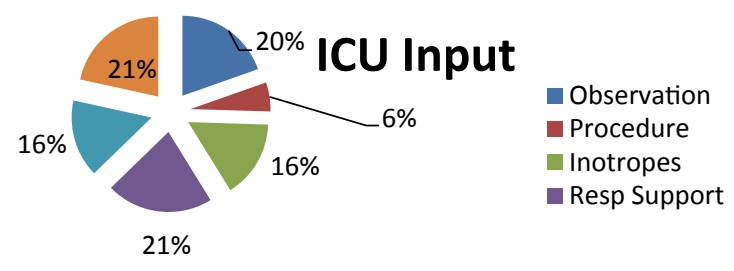

Figure 6: Chart showing the input required from ICU for trauma patients

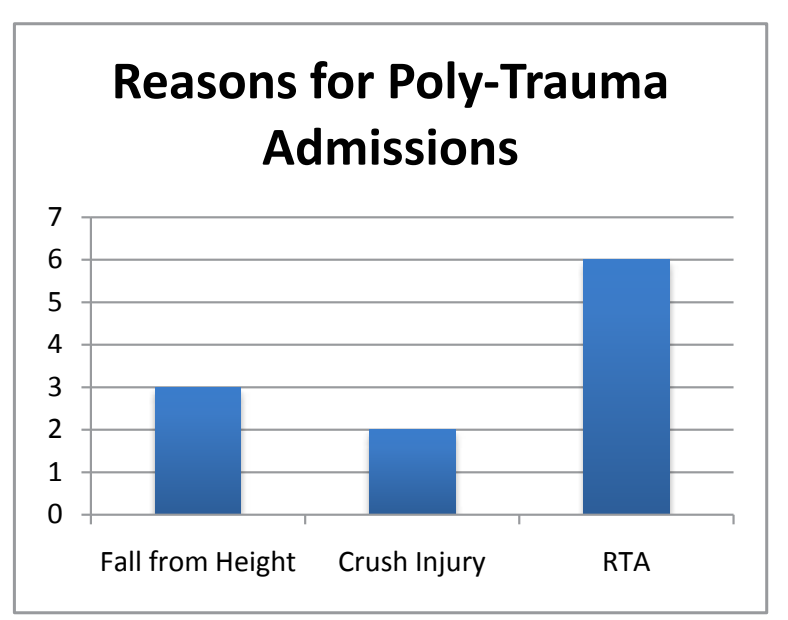

Figure 7: Chart showing the patients admitted with poly-trauma and their sustained injuries

elective orthopaedics generally involves the treatment of older patients with multiple co-morbidities and trauma with acutely injured patients, it is important to understand which patients are more likely to require intensive care post-operatively.

Figure 11 compares the total number of ICU admissions for each cohort. Unsurprisingly, almost half of the total patients [47\%] were from the "trauma" cohort. This cohort also carried the highest mortality rate with $67 \%$ of all mortalities from orthopaedic ICU admissions. Common causes included respiratory failure, acute kidney failure and septicaemia.

The average age of this cohort was 68 . A retrospective study by Tornetta et al. [9] looked at 326 elderly patients who had sustained a traumatic injury and reported a mortality rate of $18 \%$ after combined orthopaedic and general surgical operations and $9 \%$ after isolated orthopaedic operations. It is clear that older trauma patients are more unstable due to multiple co-morbidities and the severity of their injuries [10,11].

Hip fractures are associated with increased morbidity and mortality $[12,13]$. Patients sustaining hip fractures were admitted more frequently to ICU than any other trauma injury in this study. The main causes were acute renal failure, respiratory failure and hospital acquired pneumonia; a trend also reflected in the literature [14-16].

The American Academy of Orthopaedic Surgeons at their $77^{\text {th }}$ congress produced a handout on orthopaedic infections. They stated that these patients have a $60 \%$ increased risk of an ICU stay [17]. Wound and soft tissue infection combined with infected metal work represented the second largest proportion of ICU admissions from the trauma cohort. Consequently, one of the largest interventions along with respiratory support $21 \%$ was the delivery of antibiotics $21 \%$.

Approximately 75,000 hip replacements were undertaken in England and Wales last year [18]. The National Joint Registry states a mortality rate of $0.24 \%$ within 30 days which increases to $3 \%$ within two years [18]. In our study, total hip replacements were responsible for eight patients needing ICU input with a further 3 patients admitted to ICU following revision hip surgery. The majority of patients undergoing hip replacements are between 60-79 and will have associated comorbidities $[18,19]$.

The "elective surgery" cohort highlights the need for active monitoring on ICU of complex patients with co-morbidities. Figure 2 shows that $29 \%$ of patients from this cohort required close monitoring whilst $24 \%$ had cardiac problems. During total hip replacement, identifiable myocardial ischemia occurs intra-operatively, indicating myocardial strain. Coronary Heart Disease [CHD] patients are at risk of developing further complications during this type of surgery [20]. Cardiac complications after THR occur at a rate of $2 \%$ to $10 \%$ [21].

Baseline pulmonary function is a good indicator of postoperative pulmonary complications. Patients with Chronic Obstructive Pulmonary Disease [COPD] or abnormal pulmonary function tests have a $70 \%$ pulmonary complication rate [21]. As increased age causes a reduction in Forced Expiratory Volume in 1 Second [FEV1] and loss of elasticity, we can assume that patients undergoing arthroplasty surgery will have some degree of lung dysfunction [22].

General anaesthetic issues accounted for 19\% of problems in the elective cohort. General anaesthesia decreases lung compliance, depresses cough reflex, and decreases cardiac contractility. Preexisting pulmonary disease magnifies these effects. The duration of general anaesthesia also plays a role in the incidence of postoperative respiratory problems [23].

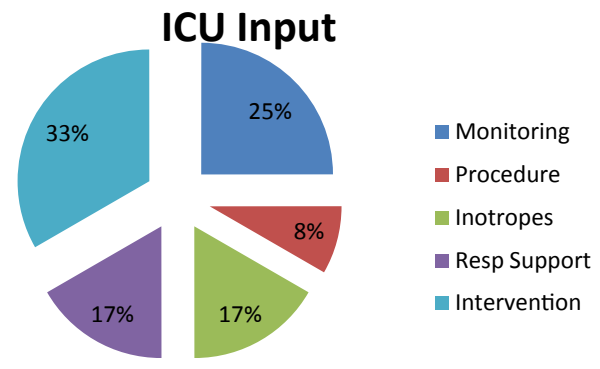

Figure 8: Chart showing the input required from ICU for poly trauma patients 


\section{Length of ICU Admission}

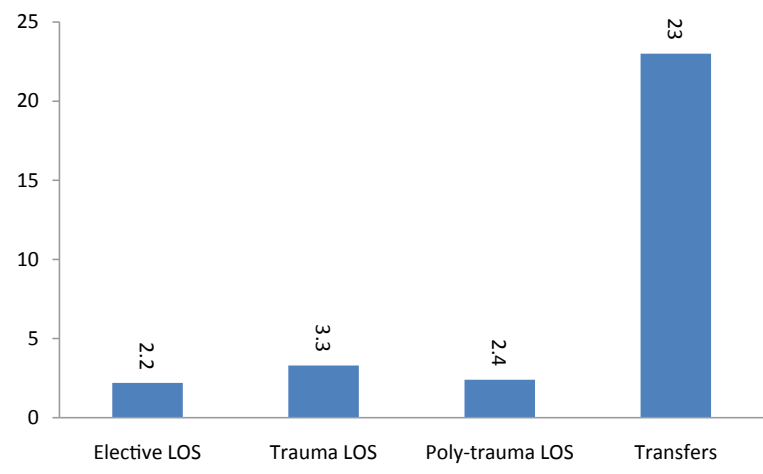

Figure 9: Chart showing the average lengths of ICU admission

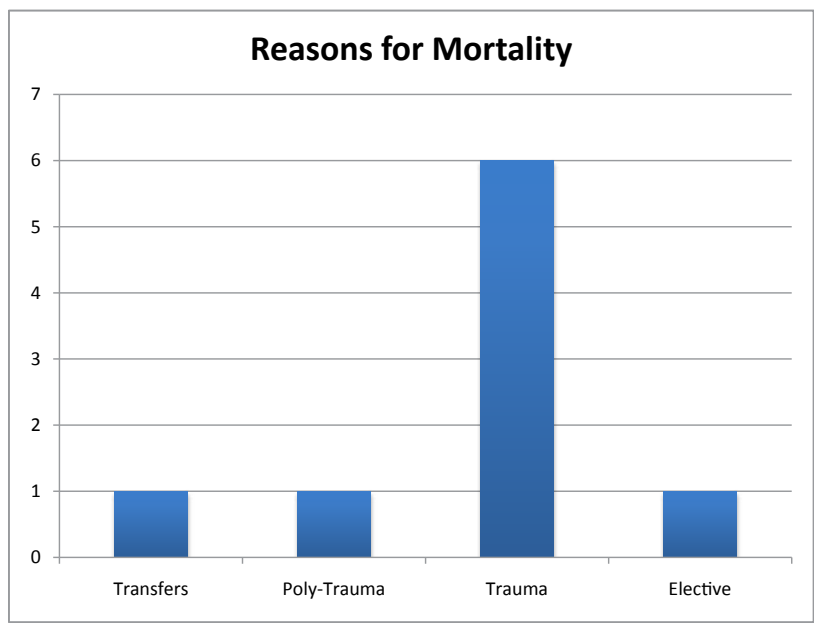

Figure 10: Chart showing the reasons for mortality following admission to ICU

$55 \%$ of patients admitted from the "poly-trauma" cohort were as a result of a road traffic accident. Monitoring for such injuries is essential due to cardio-respiratory compromise. These patients are more likely to need interventions such as chest drains and central lines when compared to their elective counterparts.

\section{Conclusion}

Intensive care units are now seen as an essential part in the treatment of critically ill surgical patients. As there is an increasing elderly population, patients often present for surgery, with pre-existing comorbidities. These comorbidities have a direct correlation on increased morbidity and mortality rates highlighting the importance of the ICU team.

The majority of orthopaedic admissions are elderly patients undergoing elective surgery or acute trauma patients. This study highlights the latter as being markedly more likely to require ICU admission postoperatively. Mortality is higher in this group with infection being a significant cause [17]. Both groups of these orthopaedic patients require a greater level of care with several possible complications, increased vulnerability, potential cardio-pulmonary compromise and requirement of general anaesthesia.

Critical care consists of 3 levels of critical care. Level 3 requires invasive ventilation, level 2 requires non-invasive ventilation and level

\section{Reason for Initial Orthopaedic Admission}

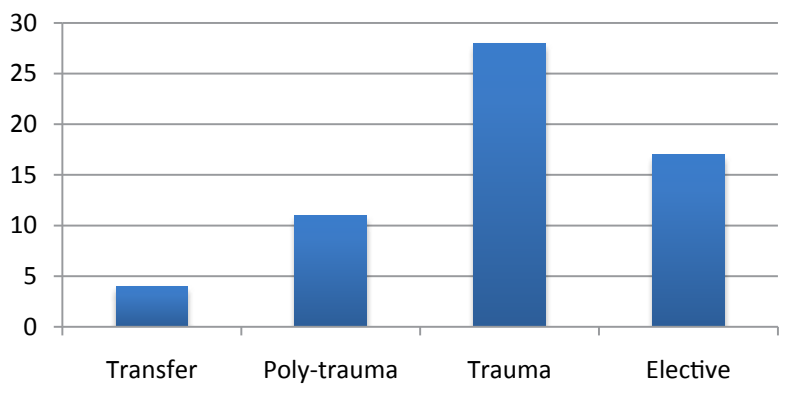

Figure 11: Chart comparing the reasons for initial orthopaedic admission

1 requires monitoring and observation. A large proportion of patients require level 1 care and $43 \%$ of patients in this study falling under that category. This asks the question of whether patients could be placed in a specific care unit specialised to provide level care to improve outcomes.

Major trauma continues to contribute substantially to high morbidity, mortality and long-term disabilities worldwide and it is therefore paramount that correct intensive care input is sought $[24,25]$.

\section{References}

1. Curran JE, Grounds RM (1998) Ward versus intensive care management of high-risk surgical patients. Br J Surg 85: 956-961.

2. (2000) The Royal College of Surgeons of England and the British Orthopaedic Association. Better Care for the Severely Injured. London: RCSEND, Professional Standards and Regulation.

3. Grossman M (1988) Introduction to Trauma Care; Philadelphia: LippincottRaven.

4. Mitchell V, Scarlett M, Amata A (2001) Trauma Admissions to the ICU of the University Hospital of the West Indies, Kingston, Jamacia. Trauma Care 2: 8689.

5. Taylor JM, Gropper MA (2006) Critical care challenges in orthopedic surgery patients. Crit Care Med 34: S191-199.

6. Nazon D, Abergel G, Hatem CM (2003) Critical care in orthopedic and spine surgery. Crit Care Clin 19: 33-53.

7. Kreis DJ Jr, Plasencia G, Augenstein D, Davis JH, Echenique M, et al. (1986) Preventable trauma deaths: Dade County, Florida. J Trauma 26: 649-654.

8. West JG, Cales RH, Gazzaniga AB (1983) Impact of regionalization. The Orange County experience. Arch Surg 118: 740-744.

9. Tornetta P 3rd, Mostafavi H, Riina J, Turen C, Reimer B, et al. (1999) Morbidity and mortality in elderly trauma patients. J Trauma 46: 702-706.

10. Moini M, Rezaishiraz H, Zafarghandi MR (2000) Characteristics and outcome of injured patients treated in urban trauma centers in Iran. J Trauma 48: 503507.

11. Duane TM, Rao IR, Aboutanos MB, Wolfe LG, Malhotra AK (2008) Are trauma patients better off in a trauma ICU? J Emerg Trauma Shock 1: 74-77.

12. Clayer MT, Bauze RJ (1989) Morbidity and mortality following fractures of the femoral neck and trochanteric region: analysis of risk factors. J Trauma 29 1673-1678.

13. Abrahamsen B, van Staa T, Ariely R, Olson M, Cooper C (2009) Excess mortality following hip fracture: a systematic epidemiological review. Osteoporos Int 20 : 1633-1650.

14. Roche JJ, Wenn RT, Sahota O, Moran CG (2005) Effect of comorbidities and postoperative complications on mortality after hip fracture in elderly people: prospective observational cohort study. BMJ 331: 1374. 
15. Handoll HH, Farrar MJ, McBirnie J, Tytherleigh-Strong G, Milne AA, et al. (2002) Heparin, low molecular weight heparin and physical methods for preventing deep vein thrombosis and pulmonary embolism following surgery for hip fractures. Cochrane Database Syst Rev CD000305.

16. Browner WS, Pressman AR, Nevitt MC, Cummings SR (1996) Mortality following fractures in older women. The study of osteoporotic fractures. Arch Intern Med 156: 1521-1525

17. American Academy of Orthopaedic Surgeons 77th Annual meeting Handout: Orthopaedic infection Prevention and Control: An Emerging New Paradigm

18. http://www.njrcentre.org.uk

19. Whittle J, Steinberg EP, Anderson GF, Herbert R, Hochberg MC (1993) Mortality after elective total hip arthroplasty in elderly Americans. Age, gender, and indication for surgery predict survival. Clin Orthop Relat Res 119-126.

20. Peters KM, Nachtsheim B, Schuster CJ, Forst R (1997) Cardiac risks in total hip replacement. Arch Orthop Trauma Surg 116: 83-87.
21. Sheppeard H, Cleak DK, Ward DJ, O'Connor BT (1980) A review of early mortality and morbidity in elderly patients following Charnley total hip replacement. Arch Orthop Trauma Surg 97: 243-248.

22. Stein M, Koota GM, Simon M, Frank ha (1962) Pulmonary evaluation of surgical patients. JAMA 181: 765-770.

23. Hole A, Terjesen T, Breivik H (1980) Epidural versus general anaesthesia for total hip arthroplasty in elderly patients. Acta Anaesthesiol Scand 24: 279-287.

24. Hofman K, Primack A, Keusch G, Hrynkow S (2005) Addressing the growing burden of trauma and injury in low- and middle-income countries. Am J Public Health 95: 13-17.

25. Park K (2000) Accidents. In Textbook of Social and Preventive Medicine. (17th edn), Banarsidas Co, India. 